Case Report

\title{
Keen's Approach : A Dynamic Approach for Zygomatic Arch Fracture Management
}

\author{
M uralee M ohan ${ }^{1}$, Tripthi P.S. ${ }^{2}$, Pratiksha Shetty ${ }^{3} \&$ Akash M enon $^{4}$ \\ ${ }^{1}$ Professor, ${ }^{2,3}$ Assistant Professor, ${ }^{4}$ Post Graduate, Department of Oral and Maxillofacial Surgery, A. B. Shetty Memorial \\ Institute of Dental Sciences, M angalore, Karnataka.
}

Correspondence: Akash Menon, S2-08, Samruddhi Residency, Kuthar, M unnru Post, M angalore, Karnataka - 575017.

Mobile: +91 9916460828, E-mail: akashmenon@ live.in

$\begin{array}{ll}\text { Received } & : 06.04 .2016 \\ \text { Review Completed } & : 12.10 .2016 \\ \text { Accepted } & : 05.11 .2016\end{array}$

Keywords : Zygomatic arch, Fracture, Intraoral approach, Gillie's technique

\begin{tabular}{|c|}
\hline Access this article online \\
\hline Quick Response Code \\
\hline
\end{tabular}

\begin{abstract}
Maxillofacial fractures are often associated with blunt head injuries of which the outward projection of the Zygomatic Arch exposes it to frequent injury. Isolated Zygomatic arch fractures account for $10 \%$ of all Zygomatic bone fractures. Depressed Zygomatic arch fractures causes restricted mouth opening due to the partial or total obstruction of the movement of mandibular coronoid and condylar processes. Numerous techniques have been used to approach the site and reduce the fractured fragments, such as the Gillie's temporal approach, Keen's intraoral approach, anterior cheek skin incision and direct open approach.

Below is a case report of an isolated Zygomatic arch fracture following a road accident in a middle aged woman that was surgically treated by open reduction through the Keen's intraoral approach. Successful reduction of the fractured segments and restoration of function was achieved with no scarring, minimal post-operative pain and satisfactory aesthetics.
\end{abstract}

\section{Introduction}

Access to the Zygomatic arch to treat fractures of the same can be achieved by various approaches. The most routinely used of these are the Keen's vestibular approach and Gillie's temporal approach, both of which are quick and simple. This article aims to demonstrate the merits of the Keen's approach with the help of a case report, and also to compare the indications of the two aforementioned approaches.

\section{Case Report}

A 42 year old female reported to the Department of Oral and M axillofacial Surgery, A.B. Shetty M emorial Institute of Dental Sciences, $M$ angalore, with a chief complaint of pain and tenderness over the right cheek bone region with reduced mouth opening.

She gave a history of Road traffic accident the previous day, with an impact to the right lateral part of the face and abrasions over the arms and legs. Clinical evaluation revealed classical signs of Zygomatic arch fracture, i.e. flattening of the face in the bird's eye view,

Depression over the Zygomatic arch region, tenderness and crepitus on palpation of the same region and a reduction in mouth opening $(22 \mathrm{~mm})$.

A CT scan (3D Reconstruction) of the head with facial cuts was ordered to confirm the clinical findings, and to visualize the fractured segments with their orientation in the axial, coronal and sagittal planes. The images depicted an $\mathrm{M}$-shaped fracture, with 2 independent segments which were medially displaced.

The fracture was classified as Group II (Arch Fracture) fracture according to Knight and North, 1961.

Open reduction of the Zygomatic arch was performed under local anaesthesia, using a Keen's intraoral approach. 


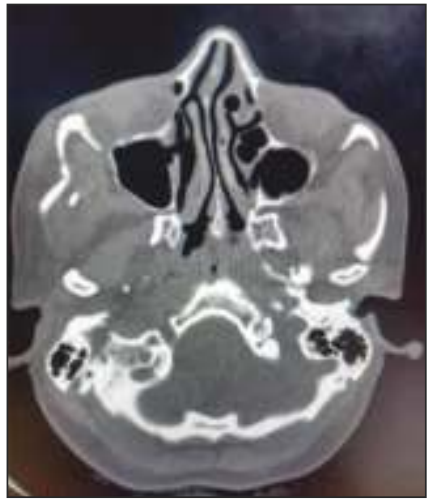

Figure 1.1

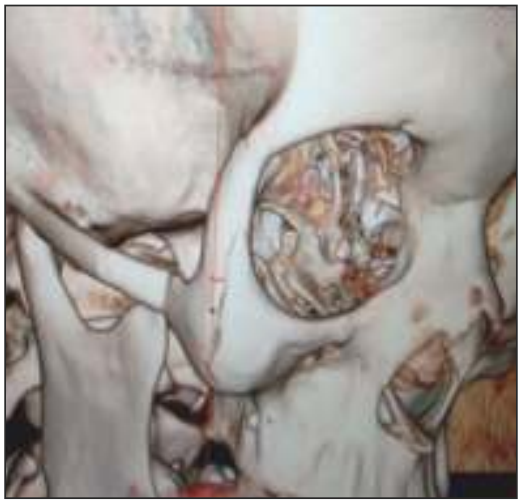

Figure 1.2

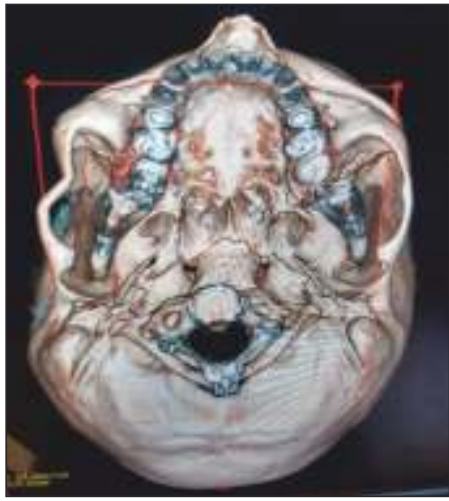

Figure 1.3

Figures 1.1 - 1.3: Preoperative Computed Tomography of the patient showing a medially displaced isolated Zygomatic arch fracture The fracture was classified as Group II (Arch Fracture) fracture according to Knight and North, 1961.

It is considered that 'less force is required by the intraoral approach than the extraoral approach, because the force is exerted where it should be, i.e. more at the centre of the fractured fragment ${ }^{12]}$.

Access was gained by an incision about $1 \mathrm{~cm}$ in length at the reflection of the upper buccal sulcus, immediately behind the Zygomatic buttress, so that a pointed curved elevator can be passed upward supraperiosteally to contact the deep or infratemporal surface of the Zygomatic bone and thus permit upward, forward and outward pressure to be exerted.

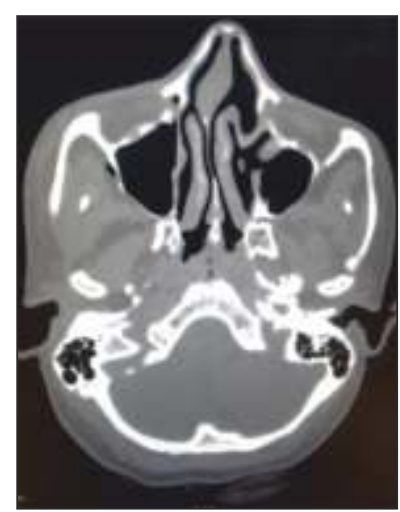

Figure 2.1

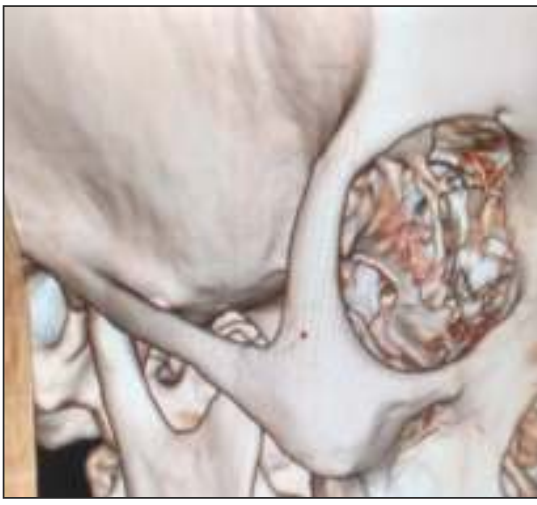

Figure 2.2
Once the elevator was in position, the surgeon applied force to lift the medially-displaced segments until a click sound was heard, indicative of the snapping back of the bone into its original position. The reduction was confirmed with digital palpation over the Zygomatic arch region, which was no longer depressed and was symmetrical with the opposite side. The intraoral incision was sutured with a resorbable suture material (4-0 Vicryl)

Post-operative CT scan revealed exact approximation of the fracture segments, with no noticeable discontinuity of the bone and bilateral symmetry.

Figures 2.1 - 2.3 : Postoperative Computed Tomography of the patient showing accurate reduction of fractured segments and bilateral symmetry

The patient was put on a standard antibiotic and analgesic regime for 1 week. Patient was recalled 10 days after the procedure and she did not have any complaints or complications in the post-operative period.

\section{Discussion}

Owing to its thin structure and susceptible position, the Zygomatic arch is quite often affected in trauma cases.
Some of the Zygomatic arch fractures are a component of complex mid-face fractures, while still others may be isolated fractures resulting from medium to low impact 
forces landing laterally on the face. When not treated properly Zygomatic arch fractures not only leads to lack of cosmesis, but also functional disorders due to pressure on the coronoid process.

The maxillary vestibular or Keen's approach, is one of the most useful approaches for open treatment of Zygomatic fractures. Access to the entire facial surface of the midfacial skeleton-from the Zygomatic arch to the infraorbital rim, to the frontal process of the maxilla-can be achieved in a relatively safe manner through this approach ${ }^{[3]}$.

The drawbacks of the other famous approach for Zygomatic arch reduction, i.e. the temporal approach or Gillie's approach, can be overcome by the Keen's approach. The middle temporal veins are often encountered when a temporal approach to the Zygomatic arch is made. The risk of haemorrhage is not great but still considerable. In the Keen's approach, virtually no anatomic hazards exist except the infraorbital neuro vascular bundle above and the posterosuperior alveolar vessels along the posterior maxilla ${ }^{[3]}$, which are seldom encountered.

Both methods are quick and simple, rarely requiring more than 15 to 20 minutes, unless fixation techniques are necessary. However, a significant difference between the two aforementioned approaches is the post-operative scarring. While the Gillie's temporal approach involves an incision within the hairline in order to mask the scar, a 2.5 cm scar will still exist regardless. Keen's approach benefits greatly from this drawback, especially in patients with high cosmetic needs. An intraoral scar hidden well within the maxillary vestibule is all that results from the buccal sulcus approach, which has higher patient acceptance.

\section{References}

1. Griffin JE, Max DP, Frey BS: The use of the C-arm in reduction of isolated Zygomatic arch fractures: A technical overview. J Craniomaxillofac Trauma 3:27, 1997

2. Rowe NL, William JL. M axillofacial injuries. Vol1. P478. Noida: Elsevier, 2009.

3. Fonseca RJ, Walker RV, Betts NJ, Barber HD, Powers MP. Oral and Maxillofacial Trauma.Vol 1. 3/e P595. Noida: Elsevier, 2009.
It should be mentioned here that, that one of the greatest advantages of the temporal approach is that it allows the application of a great amount of controlled force to disimpact even the most difficult Zygomatic fractures. This is especially useful in late treatment of fractures when partial consolidation has already taken place ${ }^{[3]}$.

Other approaches to the Zygomatic arch region such as the Lateral Eyebrow approach, Coronal approach and Lower Eyelid approach have not been discussed as they are not often used for Isolated Zygomatic arch fractures, and are associated with higher rates of morbidity.

\section{Conclusion}

Zygomatic bone, being most prominent and unique in anatomical position is highly susceptible to trauma. It also represents a primary buttress between midface and the cranium ${ }^{[4]}$. Depressed Zygomatic arch fractures generally implicate a partial or total obstruction of the movement of the condyle and of the coronoid process of the mandible, changing the opening and closure of the mouth ${ }^{[5,6]}$ Fractures of the Zygomatic arch are commonly treated by closed reduction, because the anatomical pathways of the facial nerve rule out any large incision An existing laceration in the region of the arch will be the preferred site for approach, however in its absence the Gillie's Temporal and Keen's Intraoral approach are most commonly preferred by surgeons ${ }^{[7,8]}$

While both techniques are quick, reliable, and not very technique-sensitive and have low incidence of complications, an intraoral approach is preferred when scar related cosmetics is of importance.

4. Peter Ward Booth. Maxillofacial Surgery ( $2^{\text {nd }}$ Ed.). 2007; 1:120.

5. S.J.Badjate, K.M. Cariappa. C-arm for accurate reduction of Zygomatic arch fracture-A case report. British Dental Journal, 2005; 199:275-277

6. Guven 0 . Stabilization of delayed Zygomatic arch fracture. Int J Oral Maxillofac Surg 1987; 16:445-447

7. Yamamoto K, M urakami K, Sugiura T, Fujimoto M , Inoue M, Kawakam M et al. Isolated Zygomatic Arch Fractures. J Oral M axillofac Surg 2007 